\title{
Synthesis and characterization of ganciclovir long chain lipid prodrugs
}

\begin{abstract}
Ganciclovir (GCV) is indicated for the treatment of human cytomegalo virus (HCMV) retinitis in immunocompromised patients. Sub-optimal physicochemical properties prevent GCV from reaching therapeutic concentrations in back of the eye (retina) tissue after oral and intravenous administration. Chronic high dose administration results in systemic toxicity. Local intravitreal injections suffer from poor ocular bioavailability and require repeated administration which can cause retinal detachment, retinal/vitreal hemorrhage and endophthalmitis. In the current study, we synthesized long chain acyl ester derivatives of GCV to improve lipophilicity and bioavailability. Ester conjugates (C5,C10 and $\mathrm{C} 13$ mono-and di-(O-acyl)) of GCV were synthesized in one step reaction following conventional esterification reaction. Purity of the novel prodrugs was determined with reversed phase high performance liquid chromatography. Conjugation of long lipid chain to GCV was confirmed with proton $(1 \mathrm{H})$ and carbon (13C) nuclear magnetic resonance and mass spectroscopy. Also, melting point and lipophilicity for the prodrugs and GCV were determined. MTS assay was used to assess in vitro toxicity of GCV and its long chain lipid prodrugs on human retinal pigment epithelial cell line (ARPE-19) cells. Results indicated that long chain lipid GCV prodrugs are nontoxic, safe and well-tolerated by ARPE-19 cells. These results suggest that novel long chain lipid GCV prodrugs may be further evaluated for ocular delivery and treatment of HCMV retinitis.
\end{abstract}

Keywords: characterization, cytotoxicity, 13c-nmr, ganciclovir, 1h-nmr, lipid, nmr prodrug retinal cells, synthesis
Volume I Issue 2 - 2014

\section{Kishore Cholkar, Hoang M Trinh, Aswani Dutt Vadlapudi,Ashim K Mitra}

Division of Pharmaceutical Sciences, University of MissouriKansas City, USA

\begin{abstract}
Correspondence: Ashim K Mitra, Division of Pharmaceutical Sciences, School of Pharmacy, University of Missouri Kansas City, 2464 Charlotte Street, Kansas City, MO 64108-27I8, USA Tel 8I6-235-I6I5, Fax 8I6-235-5779, Email mitraa@umkc.edu
\end{abstract}

Received: August 05, 2014 | Published: August 27, 2014

\section{Abbreviations}

AIDS, acquired immunodeficiency syndrome; $\mu$, Micro; mg, milligram; ppm, parts per million; EQ, equivalent; FDA, food and drug administration; GCV, ganciclovir; H, hour; HAART, highly anti-retroviral therapy; HCMV, human cytomegalo virus retinitis; IVT, intravitreal; IV, intravenous; $\mathrm{kV}$, kilo volts; mins, minutes; $\mathrm{mL}$, milliliter; $\mathrm{mg}$ $\mathrm{mL}^{-1}$, milligram per milliliter; $\mathrm{mg} \mathrm{kg}^{-1}$, milligram per kilogram; $\mathrm{mM}$, millimoles; $\mathrm{N}_{2}$, nitrogen Gas; $\mathrm{nm}$, nanometers; $\mathrm{S}$, seconds

\section{Introduction}

Human cytomegalo virus (HCMV) retinitis is an opportunistic infection commonly affecting naturally or iatrogenically immunocompromised patients/subjects. ${ }^{1,2} \mathrm{HCMV}$ is the leading cause of vision loss in subjects with low CD4 count $\left(<50 \mathrm{cell} / \mathrm{mm}^{3}\right)^{3,4}$ HCMV retinitis eventuates because of viral replication and induced inflammation in the retina. The infection initially develops in inner retinal layers and eventually spreads to other ocular tissues, such as retinal pigment epithelium leading to development of focal yellowish white granular patches, diffuse edema and retinal hemorrhage. All subjects identified with acquired immunodeficiency syndrome are recommended for HCMV diagnosis and immediate treatment initiation with anti-HCMV therapy. A significant reduction in HCMV prevalence has been noted with the advent of highly anti-retroviral therapy (HAART). ${ }^{5}$ However, there have been reports about HCMV relapse in AIDS patients during HAART therapy. ${ }^{6}$ Clinicians suggest that early diagnosis of HCMV retinitisin AIDS subjects and concurrent treatment with HAART and anti-HCMV therapy, with drugs such as ganciclovir (GCV), may reduce the incidence of vision loss. ${ }^{7}$ Similarly
anti-HCMV retinitis treatment with GCV is indicated for non-AIDS/ iatrogenically immunocompromised patients to prevent vision loss. ${ }^{8}$

GCV is an acyclic 2'-deoxyguanosine analogue indicated in the treatment of HCMV retinitis. ${ }^{9}$ It is the first FDA approved drug with virustatic property requiring continuous maintenance therapy to prevent HCMV relapse. ${ }^{10}$ Oral bioavailability of GCV is $\sim 5 \%$ because of its poor absorption. Due to GCV poor bioavailability, current treatment of HCMV necessitates daily intravenous (IV) infusion of GCV. ${ }^{11}$ Chronic IV administration of GCV is associated with development of systemic toxicity, poor ocular drug permeation and emergence of viral resistance. ${ }^{12,13}$ High systemic GCV dose (5-10 $\left.\mathrm{mgkg}^{-1}\right)$ is associated with side effects such as neutropenia, thrombocytopenia and abnormal hepatic function. Other raised concerns include patient's noncompliance; expensive and relative ineffectiveness. Owing to hydrophilicity of GCV, blood retinal barrier impedes deeper permeation of GCV into inner retinal tissue. Therefore, oral and IV administration of GCV cannot generate therapeutic concentrations in the back of the eye tissue (retina). There was an urgent need to avoid/minimize GCV induced systemic toxicity and improve GCV permeation. As a result intravitreal GCV inserts were developed. Local therapy mostly includes intravitreal (IVT) GCV administration $(0.2-0.4 \mathrm{mg}) .{ }^{14} \mathrm{GCV}$ has vitreal elimination halflife of $\sim 13 \mathrm{~h}$ in human ${ }^{15}$ To maintain the GCV concentration above the minimum inhibitory concentrations it necessitates frequent (2 times/week) IVT GCV administration. Chronic IVT administration is associated with vision threatening side effects such as retinal detachment, retinal/vitreal hemorrhage and endophthalmitis. ${ }^{16,17}$ Many delivery systems such as intraocular implants (biodegradable and non biodegradable), liposomes, microspheres and nanoparticles 
have been developed to reduce the frequency of GCV administration with varying degree of success. ${ }^{18-20}$

Strategies that improve GCV retinal permeation may significantly improve therapeutic efficacy and may also reduce the frequency of drug administration. Lipophilic acyl ester derivatization is a strategy to improve ocular bioavailability and permeation across cell membrane barriers. Earlier we reported synthesis, ocular disposition and antiviral efficacy of mono and di-acyl esters of small carbon chain conjugated GCV derivatives. ${ }^{21} \mathrm{GCV}$ lipid prodrugs not only improved ocular bioavailability of GCV, but the ester bond hydrolysed slowly producing sustained GCV levels. Further, it was hypothesized that conjugation of long carbon chain esters may further cause slower release of GCV. Therefore, in this communication we disclose the synthesis and characterization of long chain acyl ester conjugated GCV prodrugs.

\section{Materials and methods}

Ganciclovir (GCV) was purchased from Hubei Gedian Humanwell Pharmaceutical Co., Ltd. China. Valeric acid and 4-Dimethylaminopyridine (DMAP) were procured from Sigma Aldrich, St. Louis, MO. Dimethylformamide (DMF), N,N'Dicyclohexylcarbodiimide (DCC) and decanoic acid were obtained from ACROS Organics, NJ. Tridecanoic acid was purchased from Aldrich Chemical Co. Inc., Milwaukee, WI.

\section{Synthesis}

Long chain lipid conjugated GCV prodrugs $\left(\mathrm{C}_{10}\right.$ and $\left.\mathrm{C}_{13}\right)$ were synthesized following the conventional esterification reaction with catalytic amounts of coupling agents (DMAP, DCC) under inert $\left(\mathrm{N}_{2}\right.$ atmosphere) and anhydrous conditions. Briefly, GCV (100mg) was dissolved in anhydrous DMF by subjecting to a steam bath followed by cooling to room temperature. Hydroxyl group of GCV was activated with catalytic amounts of DMAP ( 0.2 eq.). In another flask carboxyl end group of lipid (2.5 eq.) was activated with DCC (3.0 eq.) for 30 mins. The activated lipid was added drop wise to GCV under inert conditions. The reactions were monitored for $48 \mathrm{~h}$ for the formation of prodrug with thin layer chromatography (TLC) (Scheme 1).

On the contrary, to conjugate $\mathrm{C}_{5}$ chain, valeric anhydride ( 2.5 eq.) was added to GCV. The reaction was continued for $4 \mathrm{~h}$. At the end of the reaction, $2 \mathrm{ml}$ of water was added and solvent was evaporated overnight under high vacuum (GeneVac, UK) (Scheme 1). The crude product was subjected to flash chromatography with dichloromethane and methanol (9:1) solvent system to elute the purified products. All the lipid prodrugs were recrystallized with cold diethyl ether precipitation.

\section{Characterization}

HPLC: A high performance liquid chromatography (HPLC) system comprising Shimadzu LC 20AT pump (Shimadzu, Lenexa, KS), equipped with a fluorescence detector (Perkin Elmer, Series 200 fluorescence detector, Shelton, CT) and a reversed-phase $\mathrm{C}_{12}$ column $(4 \mu \mathrm{m}, 250 \mathrm{~mm} \times 4.6 \mathrm{~mm}$, Synergy-max, Phenomenex, Torrance, CA) was utilized. Mobile phase comprised of $15 \mathrm{~mm}$ phosphate buffer (pH 2.5) and 35\% acetonitrile pumped at a flow rate of $1 \mathrm{mlmin}^{-1}$. All samples were analyzed at an excitation wavelength of $265 \mathrm{~nm}$ and at an emission wavelength of $380 \mathrm{~nm}$.

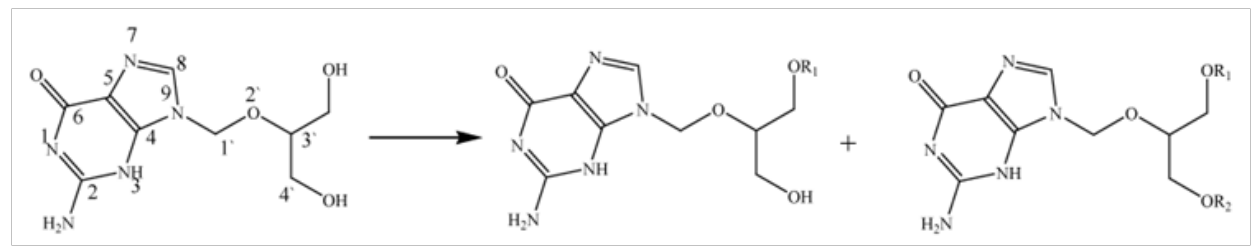

Scheme I (R)2O (valeric anhydride) (3 equiv.), DMAP (0.2 equiv.), DMF, rt, 4h, ROH (3 equiv.), DMAP ( 0.2 equiv.), DCC (3 equiv.), DMF, rt, $24 \mathrm{~h}$.

Mass spectrometry: Mass of GCV and its lipid prodrugs (monoand di-(O-acyl) conjugated)was determined with MDS Sciex API 3200 Triple Quadruple linear QTrap mass spectrometer (Applied Biosystems/MDS Sciex, Foster City, CA)by infusing the solution with a positive ion source for detection. The compounds were dissolved in HPLC grade methanol and injected at a flow rate of $15 \mu 1 \mathrm{~min}^{-1}$ into the electrospray source with a Hamilton 22 syringe pump. The mass spectra were acquired at $5 \mathrm{~s}$ per scan. The capillary temperature of $250^{\circ} \mathrm{C}$ and spray voltage of $3.5 \mathrm{kV}$ were applied. Dry nitrogen was used as sheath and auxiliary gas for analysis.

Melting point and partition coefficient: Melting point for prodrugs was determined with open capillaries using melting point instrument (MEL-TEMPII laboratory devices, USA). Briefly, a small quantity of GCV or purified lipid prodrug was transferred to glass capillary tubes and was allowed to settle at the bottom of capillary tube, by tapping. The temperature range where the solid GCV or prodrug began to melt and completely turned to liquid was recorded as melting point. Octanol/water partition coefficient was determined with ACD labs software.

NMR spectroscopy: All the synthesized prodrugs spectra were recorded on Varian $400 \mathrm{MHz}$ proton $\left({ }^{1} \mathrm{H}\right)$ and carbon $\left({ }^{13} \mathrm{C}\right)$ nuclear magnetic resonance (NMR) spectrometer (Varian, USA)in deuterated dimethyl sulfoxide (DMSO $d_{6}$ ). Tetramethylsilane (TMS) served as internal standard and the chemical shifts are expressed in parts per million (ppm) relative to TMS.A range of $5000-6000$ scans was accumulated for each spectrum.

Cell culture: In vitro cytotoxicity of novel lipid prodrugs was evaluated with Cell Titer 96® Aqueous Non-Radioactive cell proliferation assay kit (Promega, Madison, WI)on human retinal pigment epithelial cell line (ARPE-19 was purchased from American Type Culture Collection (Manassas, VA) at passage number 21). ARPE-19 was cultured and maintained as reported previously [22]. 
Briefly, ARPE-19 cells were grown in a culture medium containing DMEM supplemented with $10 \%$ (v/v) FBS (heat inactivated), $29 \mathrm{mM}$ $\mathrm{NaHCO}$, $20 \mathrm{mM}$ HEPES, $100 \mathrm{mg}$ of penicillin and streptomycin each, and $1 \%$ nonessential amino acids at $\mathrm{pH}$ 7.4. Cells were grown at $37^{\circ} \mathrm{C}$, in a humidified atmosphere of $5 \% \mathrm{CO}_{2}$ and $90 \%$ relative humidity. The growth medium was replaced with fresh medium every other day. Cells with passage number 25 were cultured in flasks and harvested at $80-90 \%$ confluency with TrypLETM Express (a superior replacement for trypsin) (Invitrogen, Carlsbad, CA, USA). Cells were then plated in 96-well plates at a density of 10,000cells/well and utilized for studies.

Statistical analysis: Data analysis for in vitro experiments was conducted at least in quadruplicate $(n=4)$ and the results are expressed as average \pm standard deviation (SD). Statistical comparison between the positive control and the experimental results was performed with Student's t-test. A $p$-value of $<0.05$ was considered to be statistically significant.

\section{Results and discussion}

\section{Synthesis}

The long carbon chain alkyl (O-acyl) mono and diesters of GCV were prepared in single step reaction following earlier described protocol from our laboratory. ${ }^{23}$ Scheme 1 shows the general method for GCV lipid prodrug synthesis. The reaction yield varied with carbon chain length. Mono (O-decyl) conjugated GCV prodrug yield was found to be higher relative to low carbon $\left(\mathrm{C}_{5}\right)$ and long carbon $\left(\mathrm{C}_{13}\right)$ chain (Table 1$)$.

Valeric acid reacted vigorously with GCV resulting in a high yield of di-(O-acyl) conjugated GCV lipid prodrug. On the other hand, the reaction yield for mono-(O-acyl) valerate $\mathrm{GCV}$ was lower. We optimized the yield to be $\sim 19 \%$ at $4 \mathrm{~h}$. On the contrary, the reactions for mono (O-acyl) decanoic $\left(\mathrm{C}_{10}\right)$ and tridecanoic acid $\left(\mathrm{C}_{13}\right) \mathrm{GCV}$ conjugates were slow. The product yield was low for di-(O-acyl) ester GCV lipid prodrug $\left(\mathrm{C}_{10}\right.$ and $\left.\mathrm{C}_{13}\right)$. In order to improve the yield for $\mathrm{C}_{10^{-}}$ and $\mathrm{C}_{13}$-di-(O-acyl) ester conjugated $\mathrm{GCV}$, the reaction was reinitiated with addition of DCC and DMAP which improved the yield with time (48h). Low carbon chain acid reactions were relatively faster resulting in diester prodrug relative to long carbon chain acids. The rationale behind synthesizing these ester prodrugs is the presence of esterases in the vitreous body ${ }^{24}$ which aid in cleaving the ester bond causing slow release of GCV. The retention factor $\left(\mathrm{R}_{\mathrm{f}}\right)$ for the novel prodrugs was determined with TLC. The $\mathrm{R}_{\mathrm{f}}$ for $\mathrm{C}_{5}$ conjugated mono- and di(O-acyl)-GCV prodrugs was 0.707 and 0.323 , respectively. $\mathrm{R}_{\mathrm{f}}$ for $\mathrm{C}_{10}$ conjugated mono- and di-(O-acyl)-GCV prodrugs were calculated to be 0.615 and 0.2323 , respectively. Similarly, the $R_{f}$ for tridecanoic acid $\left(\mathrm{C}_{13}\right)$ conjugated mono- and di-(O-acyl)-GCV prodrugs was determined to be 0.5384 and 0.108 , respectively. Ascending in carbon number lower the retention factor for both to mono- and di-(O-acyl) GCV derivatives. This result indicates that with increase in carbon number the elution of GCV prodrug is faster, i.e. di-(O-acyl) GCV prodrugs $>$ mono- $(\mathrm{O}-\mathrm{acyl}) \mathrm{GCV}$ prodrug. Within the mono- and di(O-acyl) series the trend was $\mathrm{C}_{13}>\mathrm{C}_{10}>\mathrm{C}_{5}$ indicating higher carbon number conjugated lipid prodrug eluted faster than lower carbon number GCV prodrug.

\section{HPLC, melting point and lipophilicity}

Purity of the synthesized prodrugs was determined with RPHPLC method. The purity of eluted compounds was compared with that of the parent GCV molecule. The retention times for mono- and di-(O-acyl) GCV derivatives were different. Di-(O-acyl) derivatives generated a longer retention than mono-(O-acyl) GCV lipid prodrugs. The recrystallized lipid derivatives were of high purity and the results are presented in Table 1. The melting point of the compounds was found to be lower than GCV. The melting points for the derivatives are lower with rise in conjugated carbon number. The melting point range was quite narrow indicating the purity of the product. For the mono-(O-acyl) derivatives $\left(\mathrm{C}_{5}, \mathrm{C}_{10}\right.$ and $\left.\mathrm{C}_{13}\right)$ melting point was lower than GCV. While for the di-(O-acyl) derivatives $\left(\mathrm{C}_{5}, \mathrm{C}_{10}\right.$ and $\left.\mathrm{C}_{13}\right)$ the melting points were further lower than mono- $(\mathrm{O}$-acyl $)$ derivatives $\left(\mathrm{C}_{5}\right.$, $\mathrm{C}_{10}$ and $\mathrm{C}_{13}$ ) (Table 1). These results indicate that increase in alkyl group conjugation lowered the melting points

Partition coefficient of the prodrugs was calculated with the help of ACD labs software. Results indicate that ascending carbon chain length in the diesters long lipid conjugation improved the lipophilicity of the prodrugs. There was a large increase in calculated octanol/water partition coefficient $(\log \mathrm{P})$ with conjugation of tridecanoic acid to both the hydroxyl groups of GCV (Table 1). These results suggest that the conjugation of lipid chain to GCV significantly enhances lipophilicity which may aid in improving GCV bioavailability along with slow release of GCV.

\section{Mass spectrometry}

All the long chain lipid GCV prodrugs and GCV were subjected to molecular weight analysis in positive mode with mass spectroscopy. Analysis revealed GCV and all its derivatives as proton adduct $[\mathrm{M}+1]^{+}$except $\mathrm{C}_{13}$-mono-(O-acyl) derivative which was identified as a sodium adduct $[\mathrm{M}+\mathrm{Na}]^{+}$(Table 1$)$. Figure 1 shows the broad mass spectra for $\mathrm{C}_{13}$-mono-(O-acyl) $\mathrm{GCV}$ derivative $\left(100 \mathrm{ng} \mathrm{mL}^{-1}\right)$ with a mass of 452.4 Dalton and an intensity of $7.4 \times 10^{6} \mathrm{cps}$. All theoretically calculated masses for long chain lipid derivatives were in agreement with mass spectroscopy results (Table 1), indicating that all compounds were stable.

\section{Nuclear magnetic resonance}

The proton NMR spectral data and assignments for mono- and di-(O-acyl) derivatives were calculated in comparison to the parent compound, GCV (Table 2). ${ }^{23}$ Figures 2A \& Figure 2B shows the proton NMR for GCV and GCV-C 5 conjugated lipid prodrugs. As can be seen in the spectra for GCV prodrug (Figure 2A), we do not observe any alkyl resonance peaks downstream, i.e. between $0 \mathrm{ppm}$ and $1.9 \mathrm{ppm}$. But, GCV-C $\mathrm{C}_{5}$ exhibits the proton peaks corresponding to lipid chain conjugated to hydroxyl group of GCV (Figure 2B) which on integration determined the corresponded to proton number in the lipid chain. Similar proton NMR spectra were collected for other prodrugs $\left(\mathrm{GCV}^{-\mathrm{C}_{10}}\right.$ and $\left.\mathrm{GCV}-{ }_{13}\right)$. Chemical shifts in the resonance peaks for the lipid derivatives were evident relative to GCV. Conjugation of aliphatic carbon chain $\left(\mathrm{C}_{5}, \mathrm{C}_{10}\right.$ and $\left.\mathrm{C}_{13}\right)$ deshielded proton NMR chemical shifts of carbon $(\mathrm{CH} 2 \mathrm{OCO})$ to which these groups are conjugated and the $\beta$-situated $(\mathrm{OCH})$ protons. All other resonance peaks remained same to that of GCV. Spectra were similar for mono- and di-(O-acyl) derivatives of GCV but the total number of protons calculated was double in number for di-(O-acyl) GCV lipid derivatives. ${ }^{13} \mathrm{C}$ NMR data for all prepared GCV lipid conjugates is presented in Table 2. The ester conjugated carbon (RCOO) resonance peak was evident in all the lipid prodrugs except the parent GCV molecule (Table 2). These results indicate that the long carbon chain is conjugated to hydroxyl group of GCV. 
Table I Characterization of ganciclovir (GCV) lipid prodrugs

\begin{tabular}{|c|c|c|c|c|c|c|c|c|}
\hline Drug/Prodrug & $\mathbf{R} \mathbf{I}$ & $\mathbf{R 2}$ & \%Yield & $\begin{array}{l}\text { Theoretical } \\
\text { Molecular } \\
\text { Weight }\end{array}$ & $\begin{array}{l}\text { Observed } \\
{[\mathrm{M}+\mathrm{I}]+\text { or }} \\
{[\mathrm{M}+\mathrm{Na}]+}\end{array}$ & $\begin{array}{l}\text { Melting } \\
\text { Point } \\
\left({ }^{\circ} \mathrm{C}\right)\end{array}$ & Clog P & \% Purity \\
\hline Ganciclovir* & $\mathrm{H}$ & $\mathrm{H}$ & - & 255.1 & 256.2 & $250-252$ & -0.03 & $99.5^{*}$ \\
\hline GCV-mono- $\mathrm{C}_{5}$ & $\mathrm{n}-\mathrm{C}_{4} \mathrm{H}_{9} \mathrm{CO}$ & $\mathrm{H}$ & 19 & 339.35 & 340.5 & $224-226$ & -0.1 & 97.5 \\
\hline GCV-di-C ${ }_{5}$ & $\mathrm{n}-\mathrm{C}_{4} \mathrm{H}_{9} \mathrm{CO}$ & $\mathrm{n}-\mathrm{C}_{4} \mathrm{H}_{9} \mathrm{CO}$ & 78 & 423.21 & 424.5 & $220-222$ & 1.61 & 99 \\
\hline GCV-mono- $\mathrm{C}_{10}$ & $\mathrm{n}-\mathrm{C}_{9} \mathrm{H}_{19} \mathrm{CO}$ & $\mathrm{H}$ & 27 & 409.48 & 410.5 & $216-218$ & 1.98 & 98 \\
\hline GCV-di-CIO & $\mathrm{n}-\mathrm{C}_{9} \mathrm{H}_{19} \mathrm{CO}$ & $\mathrm{n}-\mathrm{C}_{9} \mathrm{H}_{19} \mathrm{CO}$ & 70 & 563.73 & 564.7 & $198-200$ & 5.79 & 98.5 \\
\hline GCV-mono- $\mathrm{C}_{13}$ & $\mathrm{n}-\mathrm{C}_{12} \mathrm{H}_{25} \mathrm{CO}$ & $\mathrm{H}$ & 23 & 451.56 & 452.4 & $2|2-2| 4$ & 3.24 & 99.5 \\
\hline GCV-di-C 13 & $\mathrm{n}-\mathrm{C}_{12} \mathrm{H}_{25} \mathrm{CO}$ & $\mathrm{n}-\mathrm{C}_{12} \mathrm{H}_{25} \mathrm{CO}$ & 74 & 647.89 & 648.9 & $166-168$ & 5.84 & 99 \\
\hline
\end{tabular}

*Ganciclovir (GCV) purity as reported by the manufacturer (Hubei Gedian Human well Pharmaceutical Co., Ltd. China)

Table $2{ }^{13} \mathrm{C}$ NMR data for the acyl ester prodrugs of ganciclovir

\begin{tabular}{|c|c|c|c|c|c|c|c|}
\hline Carbon & GCV (23) & GCV-mono- $\mathrm{C}_{5}$ & GCV-di-C & GCV-mono- $\mathrm{C}_{10}$ & GCV-di-C 10 & GCV-mono- $\mathrm{C}_{13}$ & GCV-di-C $C_{13}$ \\
\hline 2 & 153.78 & 153.78 & 153.76 & 153.78 & 153.82 & 153.78 & 153.78 \\
\hline 4 & 151.27 & 151.27 & 151.22 & 151.27 & 151.33 & 151.27 & 151.27 \\
\hline 5 & 116.4 & 116.21 & 116.21 & 116.21 & $|16.5|$ & 116.21 & 116.21 \\
\hline 6 & 156.87 & 156.72 & 156.7 & 156.72 & 156.78 & 156.72 & 156.72 \\
\hline 8 & 137.62 & 137.56 & 137.52 & 137.56 & 137.56 & 137.56 & 137.56 \\
\hline $\begin{array}{l}\mathrm{NCH}_{2} \mathrm{O}, \\
\mathrm{I}^{\prime}\end{array}$ & 71.48 & 71.21 & 71.25 & 71.21 & 71.25 & 71.21 & 71.21 \\
\hline $\begin{array}{l}\mathrm{CH}_{2} \mathrm{OH}, \\
5^{\prime}\end{array}$ & 60.9 & 60.23 & 60.2 & 60.22 & 60.27 & 60.29 & 60.2 \\
\hline $\mathrm{CH}_{2} \mathrm{O}, 4$ & 60.9 & 63.1 & 63.07 & 63.1 & 63.1 & 63.09 & 63.1 \\
\hline $\mathrm{CHO}, 3$ & 80 & 76.79 & 76.77 & 76.79 & 76.79 & 76.7 & 76.79 \\
\hline \multirow[t]{9}{*}{ RCOO } & & 13.27 & 13.25 & 13.85 & 13.87 & 13.99 & 13.95 \\
\hline & & 17.64 & 17.66 & 22.01 & 22.02 & 22.09 & 22 \\
\hline & & 34.92 & 34.93 & 24.37 & 24.29 & 24.3 & 24.32 \\
\hline & & 35.63 & 35.64 & 28.33 & 28.39 & 28.29 & $28.4 I$ \\
\hline & & & & 28.45 & 28.47 & 28.63 & 28.72 \\
\hline & & & & 28.35 & 28.52 & 28.92 & 28.92 \\
\hline & & & & 28.64 & 28.63 & 29.04 & 29.04 \\
\hline & & & & 31.21 & 31.2 & 31.3 & 31.3 \\
\hline & & & & 33.13 & 33.13 & 33.15 & 33.15 \\
\hline RCOO & & 170.3 & 170.6 & 173 & 172.2 & 172.6 & 172.6 \\
\hline
\end{tabular}




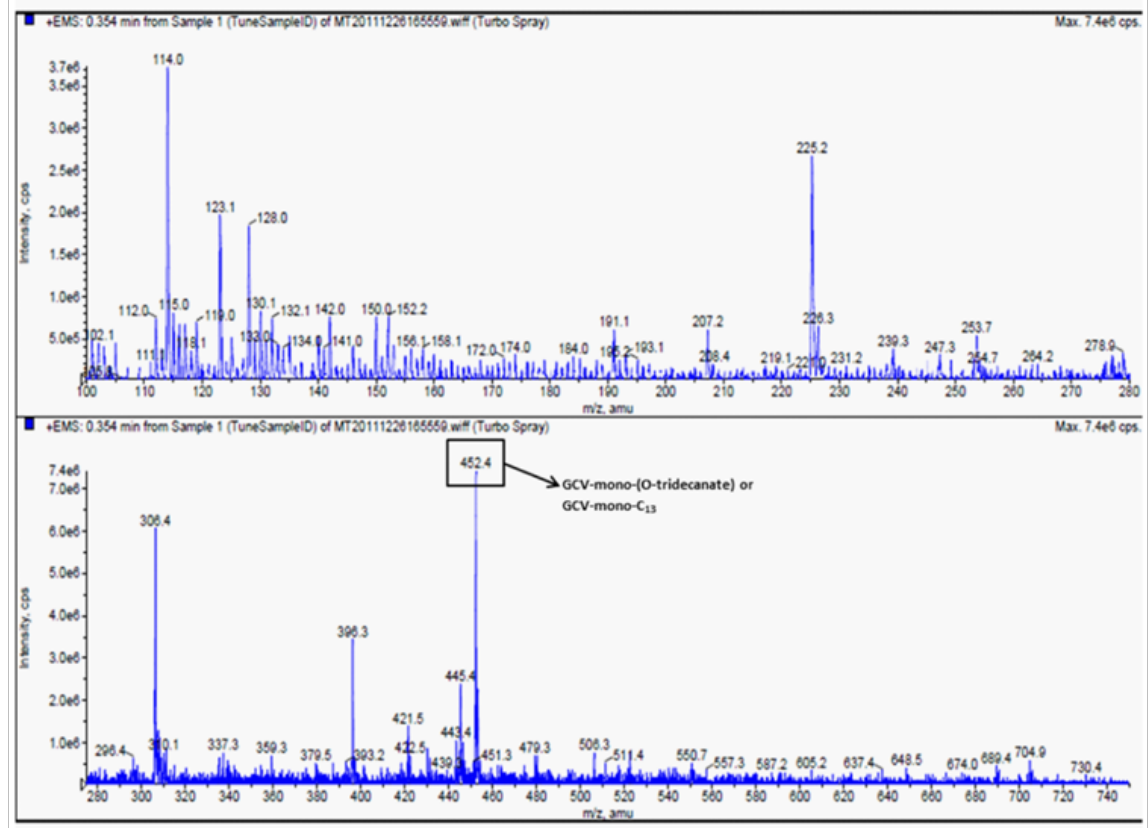

Figure I Mass spectral analysis for the purified prodrug ganciclovir-mono-(O-acyl)- $C_{13}$.

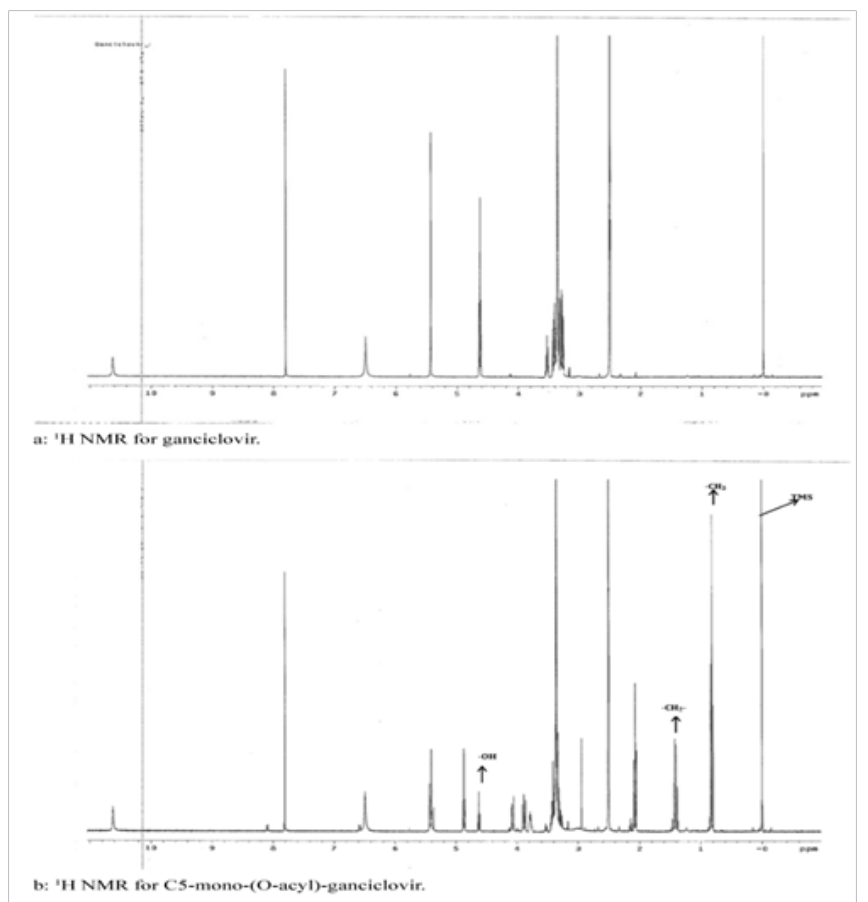

Figure 2 Proton $(\mathrm{IH})$ NMR for ganciclovir and its mono lipid conjugate.

\section{Cell culture}

To evaluate the cytotoxic effects of long chain lipid GCV prodrugs MTS assay were performed on ARPE-19 cells for $24 \mathrm{~h}$. Percent viable cells were compared with of negative control (culture medium) (Figure $3)$. DMSO (10\%) served as positive control and reduced the percent cell viable to $35 \%$. ARPE-19 cell viability after exposure to our novel long chain mono- and di-(O-acyl) GCV prodrugs was comparable to that of negative control. The result indicates that exposure of prodrugs to ARPE-19 cells for prolonged period did not induce any toxicity and the compounds were well-tolerated. Results from this study clearly suggest that our novel long chain lipid prodrugs do not cause any cytotoxicity and are safe for ocular application. 


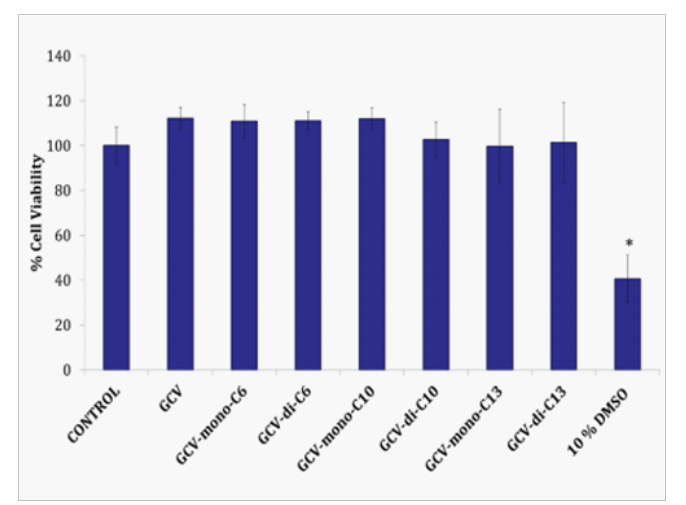

Figure 3 Ganciclovir (GCV) lipid prodrug cell proliferation studies on ARPE-19 cells. Cytotoxicity studies were conducted on ARPE-19 cells ( $N=8)$. Cells were treated with $100 \mu \mathrm{M}$ of drug/prodrug solutions for $24 \mathrm{~h}$. DMSO (10\%) served as positive control and culture medium as negative control. Statistical analysis of $p$-value $<0.05$ is considered significant and represented with symbol“*”.

\section{Conclusion}

In summary, we have successfully synthesized long chain lipid GCV prodrugs (mono- and di-(O-acyl)). These lipid prodrugs are efficiently eluted and separated with flash chromatography. HPLC purity tests demonstrated that compounds are of high purity. Also, the partition coefficient and lipophilicity number are improved with ascending in conjugated carbon chain number. Further, cytotoxicity analysis demonstrated the prodrugs to be safe and nontoxic to ARPE19 cells. These prodrugs need to be further evaluated for ocular delivery and treatment of HCMV retinitis.

\section{Acknowledgments}

This study has been supported by NIH grants R01EY09171-16 and R01EY010659-14. Also, we would like to thank Dr. Nalin Chandrasoma, Deekirikewage N. (UMKC) for his help with NMR instrument.

\section{Conflicts of interest}

Author declares that there is no conflict of interest.

\section{References}

1. Radwan A, Metzinger JL, Hinkle DM, et al. Cytomegalovirus retinitis in immunocompetent patients: case reports and literature review. Ocul Immunol Inflamm 2013;21(4):324-328.

2. Zaborowski AG. Cytomegalovirus retinitis following intravitreal triamcinolone acetonide in a patient with chronic uveitis on systemic immunosuppression. Ocul Immunol Inflamm. 2013;21(2):148-149.

3. Gallant JE, Moore RD, Richman DD, et al. Incidence and natura history of cytomegalovirus disease in patients with advanced human immunodeficiency virus disease treated with zidovudine. The Zidovudine Epidemiology Study Group. J Infect Dis. 1992;166(6):1223-1227.

4. Kuppermann BD, Quiceno JI, Flores et al. Intravitreal ganciclovir concentration after intravenous administration in AIDS patients with cytomegalovirus retinitis: implications for therapy. $J$ Infect Dis. 1993;168(6):1506-1509.

5. Stewart MW. Optimal management of cytomegalovirus retinitis in patients with AIDS. Clin Ophthalmol. 2010;4:285-299.

6. Lilleri D, Piccinini G, Baldanti F, et al. Multiple relapses of human cytomegalovirus retinitis during HAART in an AIDS patient with reconstitution of $\mathrm{CD}^{4+} \mathrm{T}$ cell count in the absence of $\mathrm{HCMV}$-specific $\mathrm{CD}^{4+}$ T cell response. J Clin Virol. 2003;26(1):95-100.
7. Yang $\mathrm{Y}$, Jiang $\mathrm{R}, \mathrm{He} \mathrm{T}$, et al. Clinical characteristic and treatment of cytomegalovirus retinitis in 80 patients with acquired immunodeficiency syndrome. Zhonghua Yan Ke Za Zhi. 2014;50(3):197-202.

8. Langner Wegscheider BJ, Ten Dam van Loon N, Mura M, et al. Intravitreal ganciclovir in the management of non-AIDS-related human cytomegalovirus retinitis. Can J Ophthalmol. 2010;45(2):157-160.

9. Razonable RR, Humar A, AST Infectious Diseases Community of Practice. Cytomegalovirus in solid organ transplantation. Am J Transplant. 2013;13(Suppl 4):93-106.

10. Lalezari JP, Friedberg DN, Bissett J, et al. High dose oral ganciclovir treatment for cytomegalovirus retinitis. J Clin Virol. 2002;24(1-2):67-77.

11. Pham PA, Bartlett JG. Ganciclovir. USA: Jones \& Bartlett learning, Burlington, MA; 2012.

12. Faulds D, Heel RC. Ganciclovir: A review of its antiviral activity, pharmacokinetic properties and therapeutic efficacy in cytomegalovirus infections. Drugs. 1990;39(4):597-638.

13. Kuppermann BD, Petty JG, Richman DD, et al. Correlation between CD4+ counts and prevalence of cytomegalovirus retinitis and human immunodeficiency virus-related noninfectious retinal vasculopathy in patients with acquired immunodeficiency syndrome. Am J Ophthalmol. 1993;115(5):575-582.

14. al-Yousuf NH, Dhillon $\mathrm{B}$, Ironside J. Ganciclovir implant: clinicopathological study. Eye (Lond). 2000;14(Pt 1):110-112.

15. Ashton P, Brown JD, Pearson PA, et al. Intravitreal ganciclovir pharmacokinetics in rabbits and man. $J$ Ocul Pharmacol. 1992;8(4):343-347.

16. Arevalo JF, Garcia RA, Mendoza AJ. High-dose (5000-microg) intravitreal ganciclovir combined with highly active antiretroviral therapy for cytomegalovirus retinitis in HIV-infected patients in Venezuela. Eur $J$ Ophthalmol. 2005;15(5):610-618.

17. Kaur IP, Kakkar S. Nanotherapy for posterior eye diseases. J Control Release. 2014.

18. Merodio M, Espuelas MS, Mirshahi M, et al. Efficacy of ganciclovirloaded nanoparticles in human cytomegalovirus (HCMV)-infected cells. $J$ Drug Target. 2002;10(3):231-238.

19. Shen Y, Tu J. Preparation and ocular pharmacokinetics of ganciclovir liposomes. AAPS J. 2007;9(3):E371-E377.

20. Bakshi NK, Fahle GA, Sereti I, et al. Cytomegalovirus retinitis successfully treated with ganciclovir implant in a patient with blood ganciclovir resistance and ocular ganciclovir sensitivity. Eye (Lond). 2012;26(5):759-760 
21. Dias CS, Anand BS, Mitra AK. Effect of mono- and di-acylation on the ocular disposition of ganciclovir: physicochemical properties, ocular bioreversion, and antiviral activity of short chain ester prodrugs. J Pharm Sci. 2002;91(3):660-668.

22. Majumdar S, Macha S, Pal D, et al. Mechanism of ganciclovir uptake by rabbit retina and human retinal pigmented epithelium cell line ARPE19. Curr Eye Res. 2004;29(2-3):127-136.
23. Gao H, Mitra AK. NMR spectral data for ester prodrugs of ganciclovir. Magnetic Resonance in Chemistry. 2000;38(8):696-700.

24. Kansara V, Balasubrahmanyan B, Mitra AK. Advancements in Ocular Drug Delivery. USA: Nova Science Publishers Inc, New York; 2007. 